This document is the accepted manuscript version of the following article:

oponowicz, E., Owen, H. L., Psoroulas, S., \& Meer, D. (2020). Geometry optimisation

of graphite energy degrader for proton therapy. Physica Medica, 76, 227-235.

https://doi .org/10.1016/j.ejmp.2020.06.023

This manuscript version is made available under the CC-BY-NC-ND 4.0

license http://creativecommons.org/licenses/by-nc-nd/4.0/

\title{
Geometry optimisation of graphite energy degrader for proton therapy
}

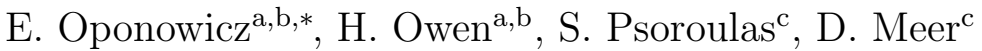 \\ ${ }^{a}$ School of Physics and Astronomy, The University of Manchester, Manchester M13 9PL, United Kingdom \\ ${ }^{b}$ The Cockcroft Institute, Daresbury Science and Innovation Campus, Warrington WA4 4AD, United \\ Kingdom \\ ${ }^{c}$ Paul Scherrer Institute, 5232 Villigen, Switzerland
}

\begin{abstract}
Introduction: Cyclotron-based proton therapy facilities use an energy degrader of variable thickness to deliver beams of the different energies required by a patient treatment plan; scattering and straggling in the degrader give rise to an inherent emittance increase and subsequent particle loss in the downstream energy-selection system (ESS). Here we study alternative graphite degrader geometries and examine with Monte-Carlo simulations the induced emittance growth and consequent particle transmission.

Methods: We examined the conventional multiple-wedge degrader used in the Paul Scherrer Institute PROSCAN proton therapy system, the equivalent parallel-sided degrader, and a single block degrader of equivalent thickness. G4Beamline Monte-Carlo tracking of protons was benchmarked against measurements of the existing degrader for proton energies from 75 to $230 \mathrm{MeV}$, and used to validate simulations of the alternative geometries.

Results: Using a careful calculation of the beam emittance growth, we determined that a single-block degrader placed close to the collimators of the ESS is expected to deliver significantly larger transmission, up to $17 \%$ larger at $150 \mathrm{MeV}$. At the lowest deliverable of $75 \mathrm{MeV}$ there is still a clear improvement in beam transmission.

Conclusions: Whilst dose rates are not presently limited on the PROSCAN system at higher energies, a single-block degrader offers the ability to access either lower energies for treatment or a larger dose rate at $75 \mathrm{MeV}$ in case transmission optimisation is desired. Single-block degraders should be considered for the delivery of low-energy protons from a cyclotron-based particle therapy system.
\end{abstract}

Keywords: energy degrader, Monte-Carlo simulations, proton therapy, graphite

\section{The Use of Graphite Degraders in Proton Therapy}

Cyclotrons are the predominant accelerator source used today for proton therapy, and isochronous variants offer access to extracted protons up to around $250 \mathrm{MeV}$ at extracted

\footnotetext{
*Corresponding author.

Email address: e.oponowicz@gmail.com (E. Oponowicz)
} 
proton currents approaching 1 microampere, with reasonable emittance suitable for modern spot-scanning treatments. One limitation of modern cyclotron designs is that they effectively offer only a single extracted energy, and so the range of energies required for patient treatment must be obtained by degrading this maximum energy (although we note the proposed designs for variable-energy extraction using either stripping [1, 2] or fixed massless septum [3]). In many cases the energy degrader is realised as a variable thickness of some robust material, and graphite is very widely used for this purpose.

It is commonly understood that degrader materials with lower atomic number $Z$ offer a smaller degree of lateral (multiple-Coulomb) scattering and energy spread for a given ionisation energy loss. Carbon $(Z=6)$ in the form of graphite is often favoured as it is straightforward to manufacture and use compared to its most-common lower- $Z$ counterparts, beryllium $(Z=4)$ and boron carbide $(Z=5,6)$. Particularly at lower energies, the lateral scattering that accompanies the ionisation slowing can be highly deleterious to the beam emittance and energy spread exiting the degrader. A system of collimators is employed to select an appropriately-small central portion of the beam, perhaps with emittances of tens of mm-mrad and an energy spread around $0.5 \%$; this in turn can restrict the overall transmission to as little as $0.1 \%$ at the lowest kinetic energies of $70 \mathrm{MeV}$, when degrading from an initial extracted proton energy of $250 \mathrm{MeV}$ [4]. On the PROSCAN system at the Paul Scherrer Institute (PSI) Centre for Proton Therapy, emittance growth and collimation place an effective lower limit of around $70 \mathrm{MeV}$ on the deliverable proton energy for which an acceptable dose rate (around $1 \mathrm{~Gy} /$ minute) can be obtained, whilst also maintaining an acceptable emittance and spot size at the patient isocentre.

There is present interest at a number of proton treatment centres in being able to deliver dose rates greatly above $1 \mathrm{~Gy} /$ minute for so-called FLASH therapy [5], and also in extending the lower limit of kinetic energy below around $70 \mathrm{MeV}$; the latter would allow a greater range of treatments - at shallower depths - to be delivered. Here we examine the role that the degrader geometry itself can play in increasing the overall beam transmission, and we will show below that a graphite degrader in the form of a single block can deliver a larger beam transmission than the conventional geometries which typically incorporate air or vacuum gaps between the parts of the absorbing material; a common geometry that is used is the so-called wedge degrader.

\section{Study of Degrader Geometries}

\subsection{The PSI PROSCAN Degrader and Alternative Geometries}

The PSI PROSCAN facility delivers protons to several treatment rooms, and presently utilises a degrader made of graphite (density $1.870 \pm 0.005 \mathrm{~g} \cdot \mathrm{cm}^{-3}$ [4]). This takes the form of a paired multi-wedge degrader (Figure 1) inserted into the beamline from opposing sides to give a uniform effective graphite thickness across the beam, and the overall degrader has entrance and exit faces which are flat and perpendicular to the beam direction; energy variation is achieved by mechanically moving the two halves in and out of the beam together, to vary the overlap and thus the total inserted absorber material. The two identical halves each comprise two full wedges $\left(23^{\circ}\right.$ wide $)$ and one half wedge $\left(11.5^{\circ}\right.$ wide $)$. This compact and 

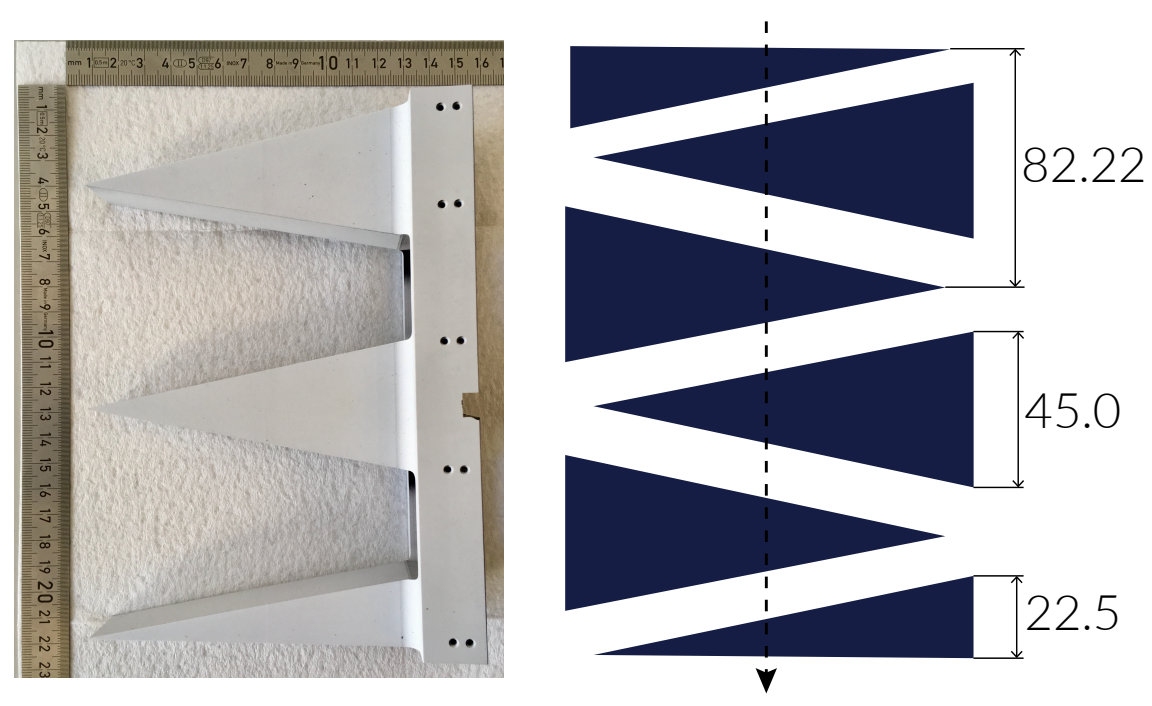

Figure 1: Left: photograph of one half of the graphite degrader (which has an alumina coating of thickness

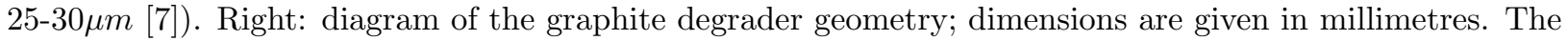
dashed arrow indicates the direction of the proton beam in the installed PROSCAN system.

lightweight wedge geometry allows for a fast energy change ( $2 \%$ energy step in $50 \mathrm{~ms}$ [4]), and the maximum thickness of interposed material is $205.55 \mathrm{~mm}$ allowing for an energy reduction from the $250 \mathrm{MeV}$ extracted from the cyclotron to a minimum of $70 \mathrm{MeV}$ [6]. The total length of the degrader including the intervening air gaps remains the same for all of the different exit energies immediately downstream of the degrader.

\subsection{Degrader Geometries}

Whilst naively one might expect that the scattering that results from a given thickness of degrader material would be independent of the degrader geometry, we postulated that the distance of the individual degrader material pieces from the downstream collimation and energy selection system might influence the overall transmission. Here we show a study of some candidate geometries to examine that hypothesis, using coupled Monte-Carlo and beam dynamic simulations of the particle transport through the degrader and the beam-optical system; our simulation model is closely representative of the real collimation system installed in the PROSCAN facility. In early operation of the PSI proton therapy system, graphite and copper blocks were used for energy reduction when delivering protons to Gantry 1 [8]; in that case, protons originated from the earlier $590 \mathrm{MeV}$ cyclotron and degraded to treatment energies over the range 85-270 $\mathrm{MeV}$ [9]. In Gantry 1 additional range shifter slabs were introduced as close as possible to the patient to minimise scattering and emittance growth.

Three geometries of graphite energy degrader were considered in this work: multi-wedge, multi-slab and single block; these are shown schematically in Fig. 2. The multiple-wedge case represents the present geometry of the degrader used in the PROSCAN system. We 
Table 1: Total thickness of graphite degrader used (irrespective of degrader geometry) in the G4Beamline simulations performed in this paper, assuming $81 \mathrm{eV}$ mean ionisation potential and $1.870 \mathrm{~g} \cdot \mathrm{cm}^{-3} \mathrm{mean}$ density. The total drift space is applicable in the multiple-wedge and multiple-slab configurations.

\begin{tabular}{ccc}
\hline Energy setting $[\mathrm{MeV}]$ & Degrader thickness $[\mathrm{mm}]$ & Total drift space $[\mathrm{mm}]$ \\
\hline 75 & 197 & 8.55 \\
100 & 179 & 26.55 \\
150 & 130 & 75.55 \\
200 & 69 & 136.55 \\
230 & 26 & 179.55 \\
\hline
\end{tabular}

also examined a degrader of equal overall length (and therefore equal air gap for a given exit energy) comprised of multiple rectangular slabs rather than wedges; we studied this because it is used in some published Monte-Carlo simulations [10] as a geometry that is considered simpler to implement yet physically equivalent to a multiple-wedge arrangement; we will confirm later in this paper that this assumption is correct. Finally, a single block degrader (with no intervening air gaps) was also examined, as it was also previously proposed as a model in simulation to predict the possible benefits of novel degrader materials such as boron carbide [11, 12. In the Monte-Carlo simulations we perform, all three geometries have a common exit face location in the overall simulated beamline, i.e. wedges and slabs have the same total length when drift spaces are included, and the block is placed at the downstream end. The total thickness of the graphite along the beam path was constant across the beam envelope and and was the same for all geometries.

The thickness of the degrader used in simulation was fine-tuned to match experimental energy measurements made at the multileaf Faraday cup in the PROSCAN beamline. This monitor is located after the multiple-wedge degrader, collimation and energy selection system (ESS). In our Monte-Carlo simulations - performed with G4Beamline based on GEANT4 the thickness adjustment required precise determination of ionisation potential and density of graphite. We use $81 \mathrm{eV}$ mean ionisation potential and $1.870 \mathrm{~g} \cdot \mathrm{cm}^{-3}$ density, and note that this value must be used in conjunction with the correct electromagnetic physics model (see below).

The resulting degrader thicknesses for different energy settings are shown in Table 1 . The $250 \mathrm{MeV}$ protons are simulated through five different degrader thickness values such that at the degrader exit they have approximate kinetic energies of 230, 200, 150, 100 and $75 \mathrm{MeV}$. This is representative of the achievable energy range (238 to $70 \mathrm{MeV}$ ).

\subsection{Simulation of Degrader Performance}

Monte-Carlo simulations were performed in G4Beamline 3.06 [13] - a beam tracking code based on GEANT4 10.05 [14] and widely used for simulations of accelerator beamlines where material absorption and scattering is encountered. A modification to the G4Beamline source code was implemented to set a custom value of the material ionisation potential. The value of ionisation potential $I_{Z}$ used is valid only in conjunction with a careful choice of the physics model and particle production range cut; here we use a small range cut 


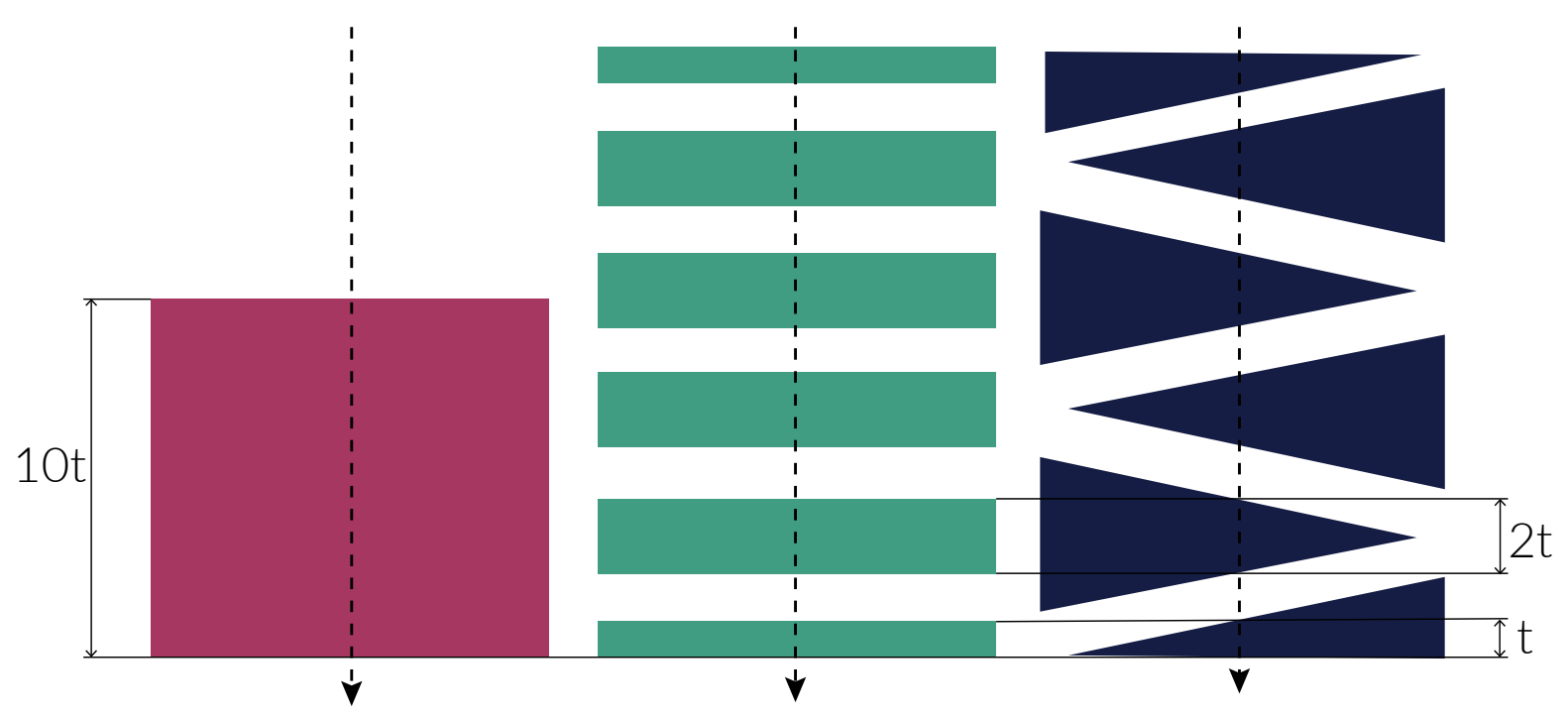

Figure 2: Sketch of three degrader geometries: single block, multiple slabs and multiple wedges. The total thickness along the reference beam path (dashed stroke) is the same for all geometries.

of $0.005 \mathrm{~mm}$. In this work we used the physics list QGSP_BIC_EMZ; option EMZ for electromagnetic processes (also called Option 4) has used the Wentzel model for Multiple Coulomb Scattering (MCS) since Geant4 10.2 release (the so-called Urban model was used previously). The Wentzel model has been shown in benchmarking studies to well describe MCS for protons in therapeutic energy ranges and in low- $Z$ materials [15]. Also, this physics list allows the use of an $I_{Z}$ for graphite of $81 \mathrm{eV}$, consistent with ICRU reference values [16].

For computational efficiency we tracked only primary protons, since secondary protons (for example, from inelastic collisions) have sufficiently-different energies and low-enough production rate that they will not pass through the collimation system and therefore do not contribute to the predicted transmission of the collimators; a realistic quadrupole aperture $(46.5 \mathrm{~mm})$ was used as a radius cut to similarly minimise the computational time. $10^{6}$ primary protons were used in all simulations to give reasonable statistical uncertainty. All beamline elements were assumed to be in vacuum as scattering within the beam pipe is insignificant. Profile monitors were placed in the beamline in their physical locations, and an additional virtual detector was placed at the exit face of the degrader to examine the beam properties before it underwent any collimation. The beam parameters at the cyclotron exit (immediately after extraction) - inferred from downstream measurements and which are used as starting parameters for beamline tracking - are listed in Table 2.

\subsection{Emittance Growth in the Degrader Beamline}

Using G4Beamline with the initial parameters described above, we examined the effect of the degrader geometry on the expected emittance. Here we focus on the beamline 
Table 2: Beam properties at the reference cyclotron extraction point used in G4Beamline simulations; relevant properties are one- $\sigma$ values.

\begin{tabular}{lll}
\hline Kinetic energy & & $250 \mathrm{MeV}$ \\
Energy spread & & $0.09 \%$ \\
\hline \multirow{3}{*}{ Horizontal } & size & $1.33 \mathrm{~mm}$ \\
& divergence & $1.06 \mathrm{mrad}$ \\
& emittance & $1.31 \mathrm{\pi mm} \cdot \mathrm{mrad}$ \\
& Twiss $\alpha$ & -0.38 \\
& Twiss $\beta$ & $1.34 \mathrm{~m}$ \\
\hline \multirow{4}{*}{ Vertical } & size & $2.13 \mathrm{~mm}$ \\
& divergence & $0.92 \mathrm{mrad}$ \\
& emittance & $1.75 \mathrm{mmm} \cdot \mathrm{mrad}$ \\
& Twiss $\alpha$ & -0.49 \\
& Twiss $\beta$ & $2.57 \mathrm{~m}$ \\
\hline
\end{tabular}

Table 3: Properties of the circular-aperture collimators KMA3-KMA5 in the PROSCAN beamline.

\begin{tabular}{cccc}
\hline Name & Length $[\mathrm{mm}]$ & Entrance radius $[\mathrm{mm}]$ & Exit radius $[\mathrm{mm}]$ \\
\hline KMA3 & 75 & 3.5 & 5.14 \\
KMA4 & 125 & 6.5 & 13.0 \\
KMA5 & 75 & 15.0 & 15.85 \\
\hline
\end{tabular}

from the cyclotron reference extraction point up to the energy selection system (ESS), as shown schematically in Fig. 3. Following the reference cyclotron exit point there are three quadrupoles (QMA1 to QMA3); In daily operation these quadrupoles have their strengths adjusted for each desired energy after the degrader (as explained later in 4.2). However, as minimum emittance growth is obtained for the beam waist at the degrader centre [17, in our studies here the strengths of QMA1-QMA3 were fixed for all energies to ensure maximum possible beam focusing at the degrader. Subsequently, protons enter the degrader and then pass through a collimation system (KMA3 to KMA5). KMA3 and KMA5 are copper collimators with selectable apertures; in these studies constant circular apertures were used in both cases (apertures given in Table 3). KMA3 is followed by KMA4, a carbon collimator with a fixed circular aperture. The last two beam optic elements before the beam enters the energy selection system are quadrupoles QMA4 and QMA5. Several beam profile monitors served for benchmarking G4Beamline simulations against measurements: MMAP1/2 located after the cyclotron exit, MMAP5/6 before the degrader entrance, MMAP9/10 after the first two collimators, and MMAP11/12 at the end of the beamline section studied here. The monitors used are air-actuated, insertable multi-strip ionisation chambers (MSIC) with either $1 \mathrm{~mm}$ or $4 \mathrm{~mm}$ strip pitch (over the beam sizes being measured), and have been previously presented in detail in the literature [18, 19]; the effective resolution of the strips is taken account of in the analysis below. 


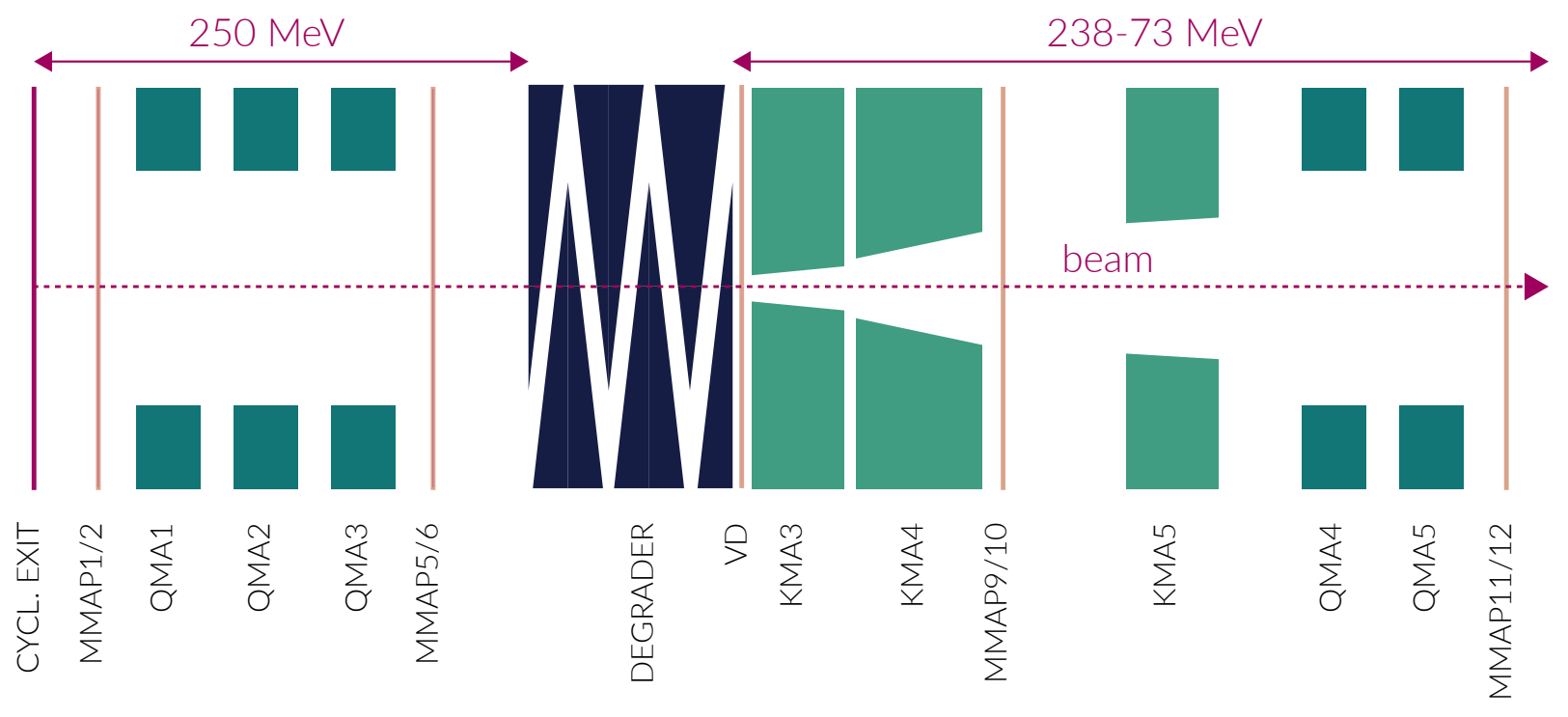

Figure 3: Schematic diagram of the initial part of the PSI PROSCAN beamline considered in this work: MMAP - profile monitors, QMA - quadrupoles, VD - virtual detector, KMA - collimators. The elements are not to scale.

Table 4: Different strengths of QMA4 for benchmarking beam profile measurements at $200 \mathrm{MeV}$ energy setting. A negative strength value indicates a horizontally-defocusing quadrupole (D-quad).

\begin{tabular}{cc}
\hline Ratio (w.r.t. standard setting) & Strength $[\mathrm{T} / \mathrm{m}]$ \\
\hline $100 \%$ & -9.172 \\
$110 \%$ & -10.082 \\
$120 \%$ & -10.992 \\
\hline
\end{tabular}

\subsection{Benchmarking}

To validate the G4Beamline simulations against the experimental data, beam profile measurements were compared for standard beamline settings. Beam position distributions in both horizontal and vertical planes at monitor MMAP5/6 - before the beam enters the energy reduction system - are shown in Fig. 4. We also modified the strength of quadrupole QMA4 (Table 4) to observe whether these changes have a consistent impact on the beam profile at the following monitor MMAP11/12, in both simulation and experiment. As shown in Fig. 5, we assessed that the simulations are in good agreement with the measurements and well represent the beamline. The discrepancies between the two come from statistical limitations of Monte-Carlo simulations and day-to-day fluctuations of the beam parameters, which are similar to the differences observed in simulations.

\subsection{Emittance Determination}

After a proton beam has passed through a degrader and lost significant energy, its emittance and energy spread is defined for the downstream use (normally patient treatment at 

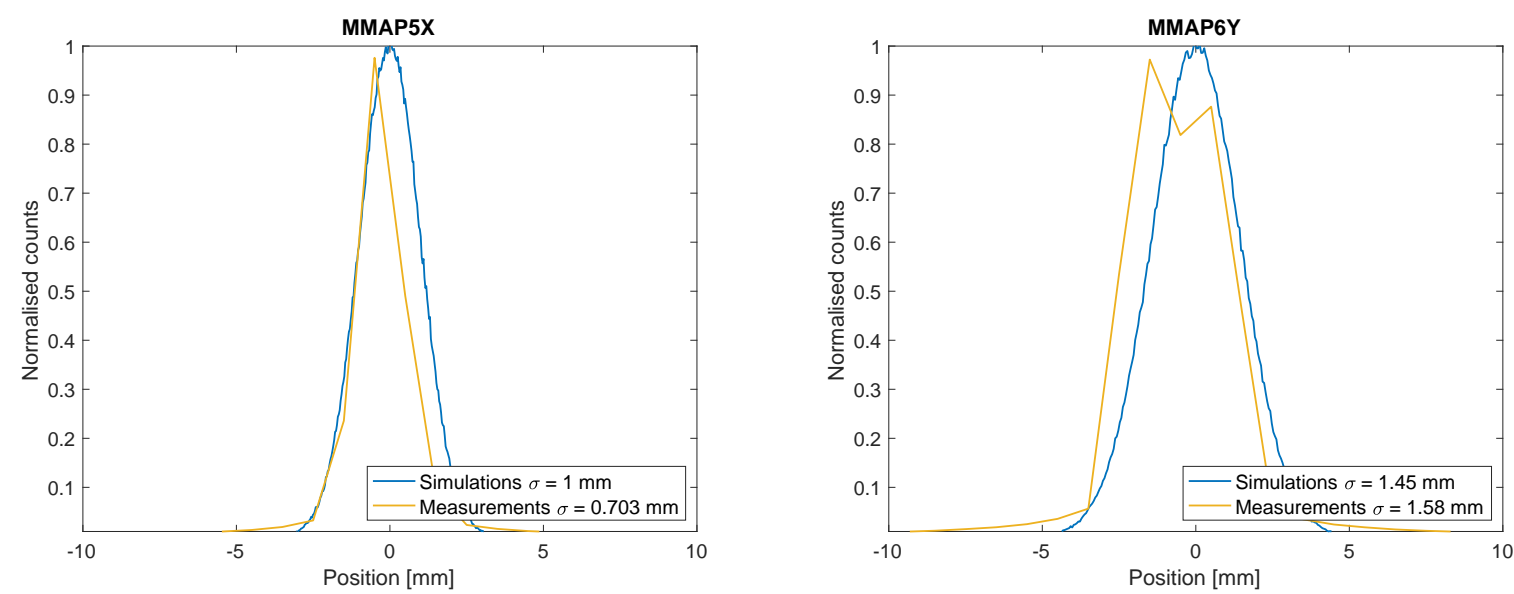

Figure 4: Horizontal (left) and vertical (right) beam profiles at monitor MMAP5/6 before the degrader entrance (monitor has $1 \mathrm{~mm}$ pitch strips over the measured beam size [18, 19]); measurements (yellow) and simulations (blue). The beam energy is $250 \mathrm{MeV}$ at this location.
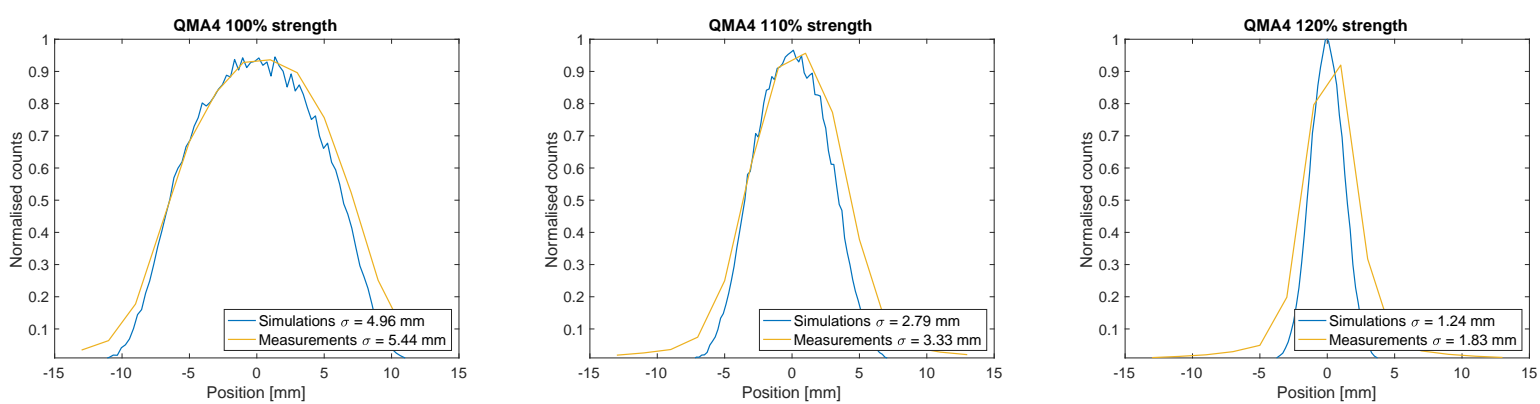

Figure 5: Vertical position distribution at monitor MMAP11/12 for $200 \mathrm{MeV}$ beam and for example quadrupole strength variations (monitor has $4 \mathrm{~mm}$ pitch strips over the measured beam size [18, 19]); measurements (yellow) and simulations (blue). The installed multiple-wedge degrader was inserted into the beamline in both simulation and experiment. 
the gantry exit) by emittance and energy collimators. To maintain a combination of reasonable spot size and energy spread at the patient, typical values for the selected emittance are a few tens of mm-mrad. However, we are interested in the fraction of particles which can be directed into that collimation system, and hence we must determine the emittance arising from the degrader itself. In simulation, we place a virtual detector at the degrader exit to observe this.

Interactions from different processes take place when a proton beam passes through matter; these include both electromagnetic and nuclear effects. Due mainly to the inelastic nuclear interactions, an initially Gaussian distribution of particles in both angle and position acquires non-Gaussian tails in phase space [20]. Here, we wish to obtain a reliable estimate of the emittance of the core of the beam particles, as this is a good measure of what emittance will be transmitted to the patient and hence is a good predictor of the spot size. We note that non-Gaussian distributions are often encountered in degrader calculations, and a simple determination of the r.m.s. emittance - using all the outgoing particles - can be highly sensitive to the number of tail particles; it is not a reliable estimate of the core emittance that arises mostly from multiple Coulomb scattering. Instead, we adapt the method of Tesse et al. [12] to obtain the beam core emittance, which iteratively removes the non-Gaussian tail particles from the sample distribution; since the tail particles are at larger amplitudes they are not transmitted through the collimators, and so this method gives a relevant emittance estimate. We apply it here to the particle distribution at the degrader exit face.

We start by defining a reduced angle for each particle exiting the degrader as

$$
\tilde{\theta}=\left(\frac{\theta_{x}-\bar{\theta}_{x}}{\sigma_{\theta_{x}}}\right)^{2}+\left(\frac{\theta_{y}-\bar{\theta}_{y}}{\sigma_{\theta_{y}}}\right)^{2},
$$

where $\theta_{x}$ is the horizontal angle and $\bar{\theta}_{x}$ and $\sigma_{\theta_{x}}$ are the mean and standard deviation values of the angular distribution in the horizontal plane; a similar expression is used for the vertical plane. If $k$ random variables are independent and all follow normal distributions, the sum of their squares follows a $\chi^{2}$ distribution with $k$ degrees of freedom. Here, the horizontal and vertical angles are independent. Those particles with large angles are then progressively removed until the reduced angular distribution follows a $\chi^{2}$ distribution with two degrees of freedom (Fig. 6). Next, we perform the same procedure to obtain a reduced position distribution. Having removed the tail particles the resulting beam phase space is Gaussian (Fig. 7) and we may then reliably calculate an r.m.s. emittance from it.

The emittance is evaluated using the statistical definition for the $2 \mathrm{D}$ trace space coordinates $w$ and $\theta_{w}$ - the particle's position and angle respectively - which both have a Gaussian distribution. The emittance of the entire distribution of $n$ particles is expressed as

$$
\epsilon=\sqrt{\left\langle w^{2}\right\rangle\left\langle\theta_{w}^{2}\right\rangle-\left\langle w \theta_{w}\right\rangle}
$$



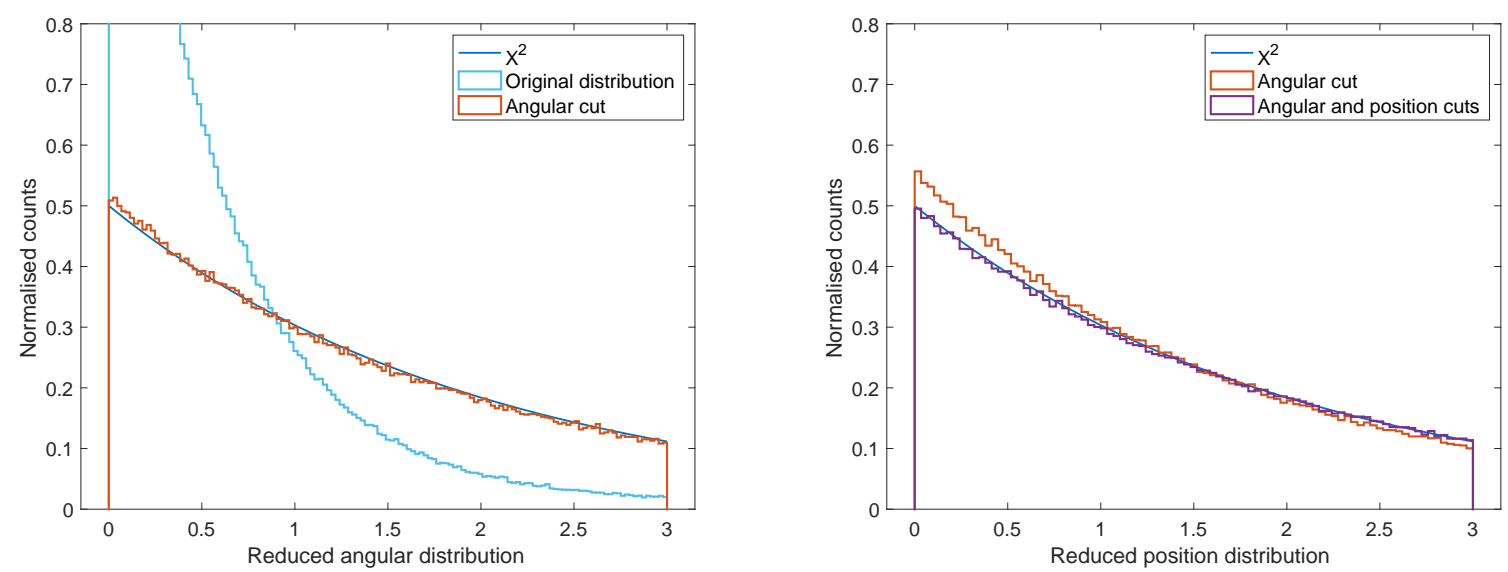

Figure 6: Two steps to obtain the Gaussian core of the beam in angle and position. Normalised counts as a function of reduced angle are shown on the left: the original distribution (blue) and the resulting distribution after a cut in reduced angle (orange) which follows a $\chi^{2}$ function with two degrees of freedom. Normalised counts as a function of reduced position are shown on the right: the distribution resulting from the reduced angle cut (orange) and the distribution after making a cut in reduced position (purple) that follows the smooth $\chi^{2}$ function.
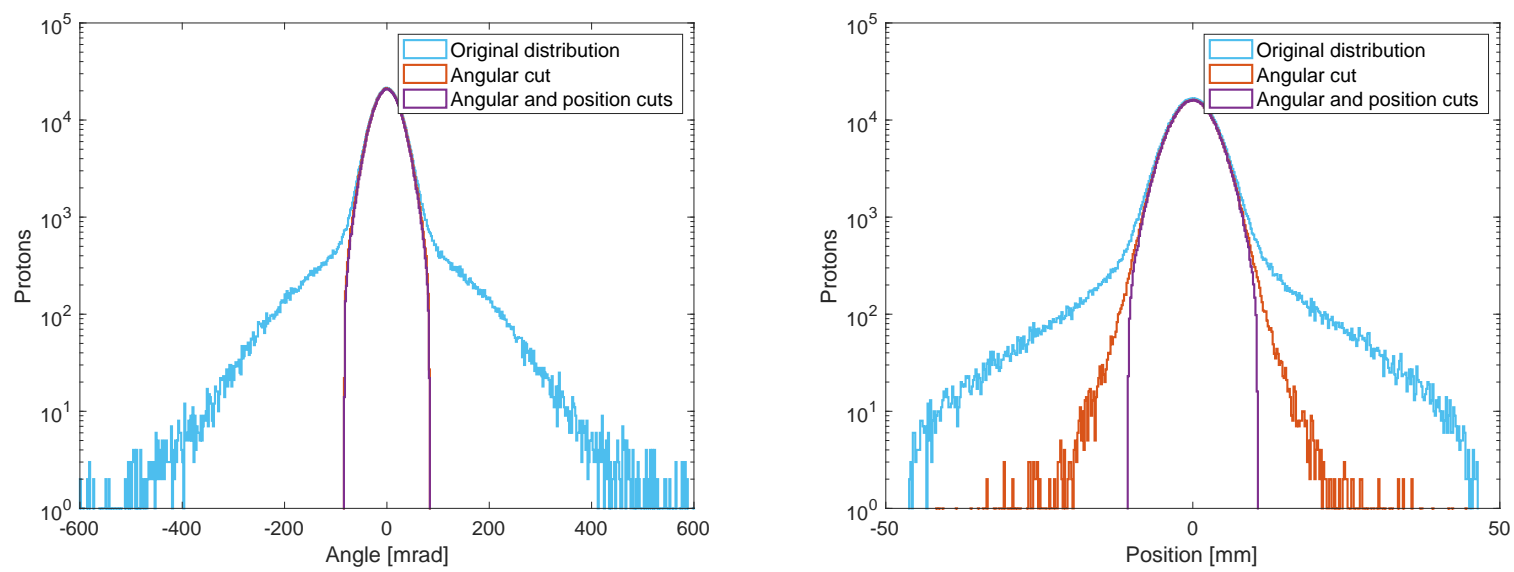

Figure 7: Impact of the beam core extraction method on the horizontal angle (left) and position (right) distributions at the degrader exit for a $150 \mathrm{MeV}$ proton beam. Without the removal of the tail particles the estimate of the r.m.s. emittance is highly sensitive to the tail particles and in particular to statistical fluctuations in the number of those particles. After tail removal the emittance estimate more reliably reflects the core emittance which passes through the ESS and downstream beam transport. 
Table 5: Benchmarking the method of beam core extraction: properties are shown of the $150 \mathrm{MeV}$ beam at monitor MMAP11/12 when calculated from two different simulations. On the left is shown the 'Full Run' in which no cuts in the particle tails are made at the exit of degrader; only the effect of the subsequent collimators is experienced by the protons. On the right is shown the 'Two-Step Run' in which the tail particles have been removed according to the iterative Tesse method. This method gives a reliable emittance at the degrader exit, but replicates the overall transmission and emittance downstream of collimation system; the two cases have the same predicted values within the statistical uncertainties from Monte-Carlo errors.

\begin{tabular}{lrr}
\hline Parameter & Full Run & Two-Step Run \\
\hline Transmission [\%] & $4.74 \pm 0.02$ & $4.75 \pm 0.01$ \\
$\epsilon_{x}[\mathrm{~mm} \mathrm{mrad}]$ & $29.08 \pm 1.53$ & $28.27 \pm 0.50$ \\
$\epsilon_{y}[\mathrm{~mm} \mathrm{mrad}]$ & $18.94 \pm 0.59$ & $18.57 \pm 0.23$ \\
\hline
\end{tabular}

where

$$
\begin{aligned}
\left\langle w^{2}\right\rangle & =\frac{1}{n} \sum_{i=1}^{n}\left(w_{i}-\langle w\rangle\right)^{2}, \\
\left\langle\theta_{w}^{2}\right\rangle & =\frac{1}{n} \sum_{i=1}^{n}\left(\theta_{w_{i}}-\left\langle\theta_{w}\right\rangle\right)^{2}, \\
\left\langle w \theta_{w}\right\rangle & =\frac{1}{n} \sum_{i=1}^{n}\left(w_{i}-\langle w\rangle\right)\left(\theta_{w_{i}}-\left\langle\theta_{w}\right\rangle\right) .
\end{aligned}
$$

If only a fraction $F$ of the total number of particles is enclosed in the contour defining the emittance $\epsilon_{F}$ [21], then

$$
\epsilon_{F}=-2 \epsilon \cdot \ln (1-F)
$$

\subsubsection{Validation of the Method}

To validate the method of cutting the non-Gaussian tails to determine the emittance, we performed a number of Monte-Carlo simulation runs. Results of full runs from the cyclotron exit to MMAP11/12 are compared with runs consisting of two steps. In the first step the beam was tracked until the energy degrader exit, where the tail particles were removed. The resulting core of the beam was then used in the second step as input beam transported from the degrader exit to monitor MMAP11/12. The transverse emittance and transmission of the beam for the two cases are presented in Table 5 which confirms the correctness of the method.

\section{Effect of Degrader Geometries}

\subsection{Transverse Emittance}

The r.m.s. emittance was calculated at two different locations in the beamline. The $\chi^{2}$ cut method described in 2.6 was applied first to the particles passing the virtual detector (at the degrader exit, Fig. 8) where the beam is not yet collimated. Horizontal and vertical emittances were determined for different degrader thicknesses and geometries. The Tesse 

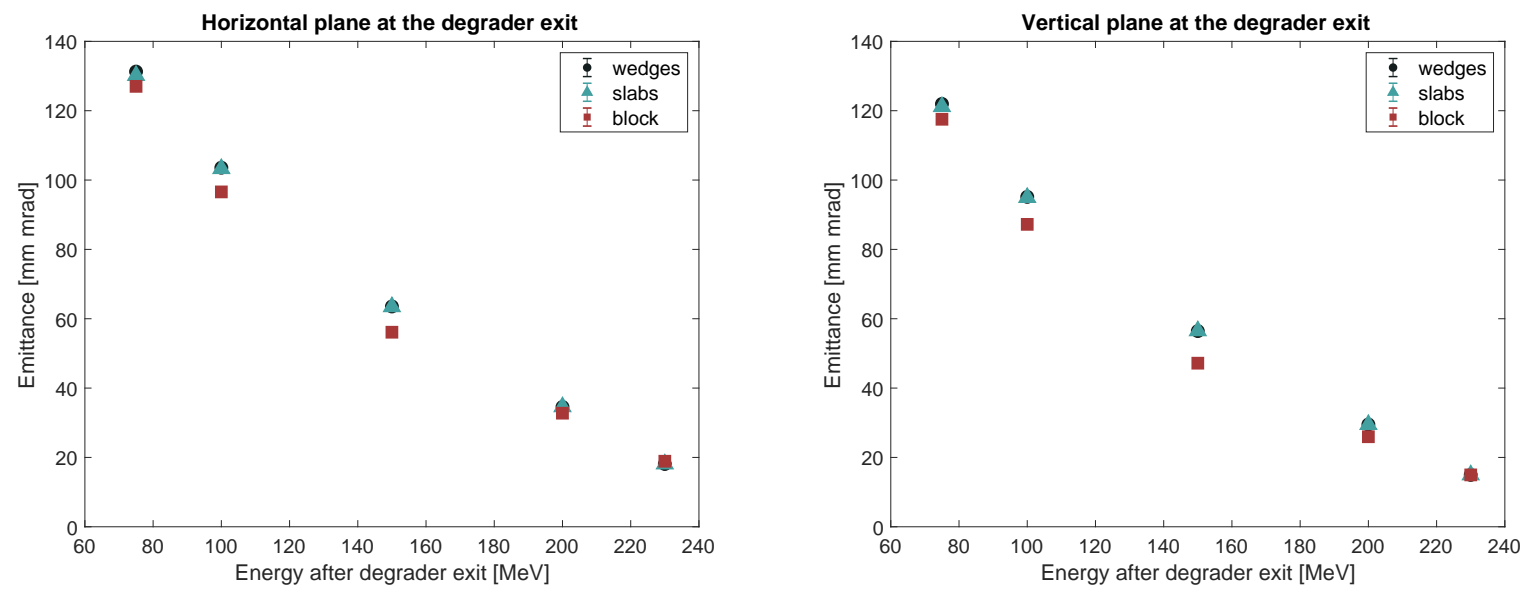

Figure 8: Horizontal (left) and vertical (right) beam emittance at the exit face of the degrader for different energy settings and various geometries. The error bars show the statistical (Monte-Carlo simulation) uncertainties and are small for some data points. There is a clear reduction in emittance when the 'block' geometry is used.

procedure of cutting off the large position/angle particles removes most of the numerical uncertainties. As would be expected, at higher beam energies (smaller thickness of degrader) the exit emittance is smaller; the beam emittance is minimum for the solid block case.

The second location is at the monitor MMAP11/12 after the degrader and after the collimation system (Fig. 9). Here, the beam distribution is Gaussian due to the action of the collimators and hence there is no need to apply the Tesse method; no significant difference can be seen between three geometries. This is due to the fact that the fraction of primary particles which lie in the core emittance is different for the three geometries and this fraction is transmitted downstream (Fig. 10).

\subsection{Beam Transmission}

Particle transmission is here defined as the fraction of particles that pass to MMAP11/12 (Fig. 10 compared to the original number at the cyclotron extraction reference point. There is a significant improvement in the transmission of the beamline with the single-block energy degrader, as compared to multiple wedges or slabs (which are similar to each other); up to $17 \%$ more protons are predicted to be transmitted if a single degrader block is used.

\section{Discussion}

\subsection{Single Block vs Multi-wedge Geometry}

The particle transmission from the cyclotron to the monitor MMAP11/12 is predicted to be higher when a single block degrader is used. Our explanation for this is that the scattering material in the single-block case is nearer to the collimators than when a multiple-wedge system is used; whilst the small-angle spread from the different geometries is the same, in the single-block case the angular spread is generated over a smaller longitudinal thickness, 

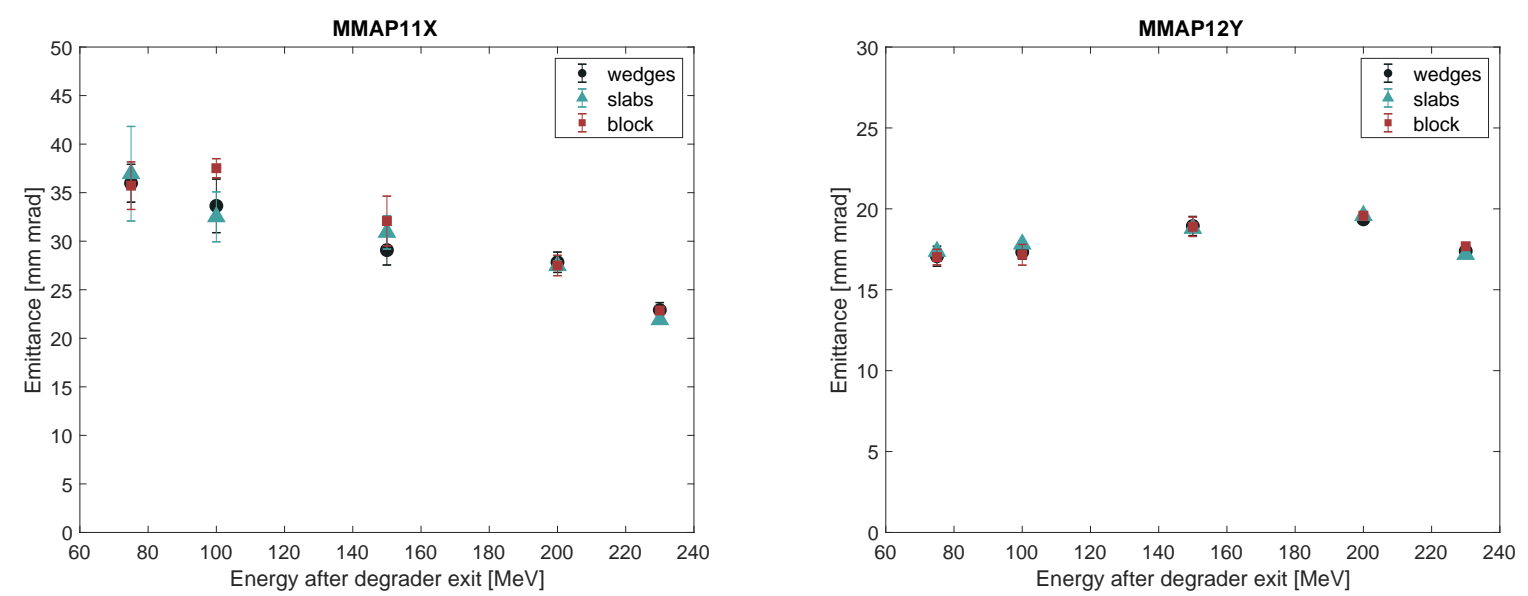

Figure 9: Horizontal (left) and vertical (right) beam emittance at profile monitor MMAP11/12 for different energy settings and various degrader geometries. The three geometries are equivalent.

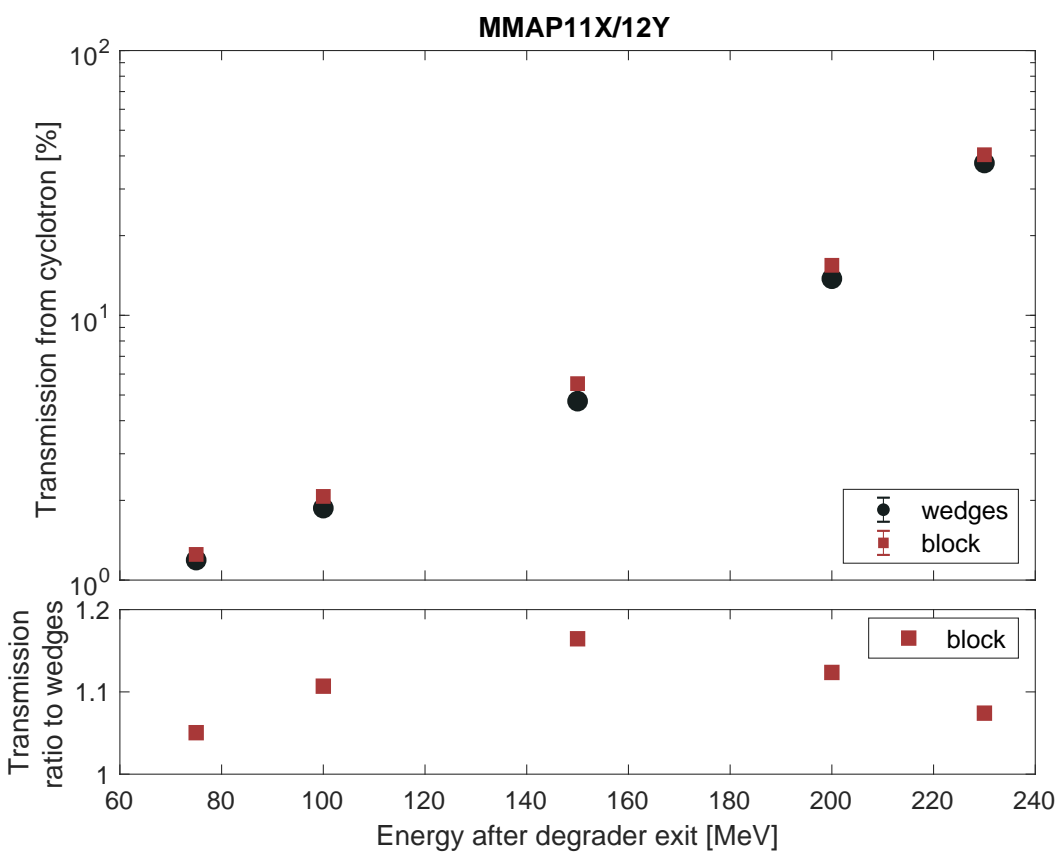

Figure 10: (Top) particle transmission from the cyclotron to monitor MMAP11/12 for different energy settings and various degrader geometries (top); (bottom) ratio of the particle transmission for the single block geometry to the transmission through the conventional multi-wedge degrader at MMAP11/12. The relative transmission peaks at $150 \mathrm{MeV}$ are probably due to the relative inelastic scattering rate reaching a maximum at this energy 10 . 
reducing the generated emittance and allowing a larger transmission to be obtained. Hence, beam losses are predicted to be lower over the entire operating energy range when a degrader/ESS is equipped with a block degrader. Since the transmission is higher, this would allow a lower energy to be reached using a single-block degrader. It also implies that a higher-density material used as a single block would be advantageous, since the scattering encountered alongside the ionisation loss would be located in as small a longitudinal distance as possible. This is postulated to be an advantage for materials such as boron carbide, which have a larger density than graphite. The disadvantage is that single-block methods likely necessitate the use of a rotating set of fixed-thickness blocks rather than the continuous adjustment possible with the wedge degrader method.

The use of a single-block degrader may be advantageous for extending an acceptable dose rate below the $70 \mathrm{MeV}$ lower limit presently assumed today at many centres. A single-block degrader is also favorable when using high-density materials such as boron carbide if there are stringent space requirements; for example, in modern compact gantry designs - that incorporate an energy degrader onto the gantry itself - there is restricted space between the magnetic elements and so a more compact degrader system is to be preferred. There are, however, beam transfer situations for which high beam transmission is not the primary goal, for example cyclinac solutions for proton computed tomography [22].

\subsection{Intensity Compensation}

Another example when the beam losses are purposely introduced to the system are beamlines with an intensity compensation scheme. In the PSI PROSCAN facility a multi-wedge degrader gives a rapid energy change, and the analytical calculations in Anferov et al. [17] concluded that a double beam waist (i.e. in both $x$ and $y$ ) located in the degrader centre of mass gives minimum emittance growth and maximum transmission. However, after natural ratio of intensities transmitted through the ESS can be as high as $I_{250 \mathrm{MeV}} / I_{70 \mathrm{MeV}} \sim 10^{3}$ across the energy range. Operationally, this ratio is reduced to $\sim 10$ at the patient by using intensity compensation [7, as follows. A beam waist at $70 \mathrm{MeV}$ is made to maximise transmission at this lowest energy, and the same quadrupole settings are used at higher energies; the focusing is no longer optimal but the transmission naturally rises as the scattering loss goes down. These are the quadrupole settings used in the present study. However, in real operations the beam transmission at energies above $150 \mathrm{MeV}$ is actually too high, and so from $150 \mathrm{MeV}$ to $250 \mathrm{MeV}$ the beam spot is deliberately defocused - both in front of the degrader and after the ESS - to induce extra loss [23].

We can quantitatively compare the effects of different beam focusing at the degrader entrance upon the particle transmission through the PSI beamline, using a $150 \mathrm{MeV}$ energy for illustration. Table 6) shows the difference in transmission at $150 \mathrm{MeV}$ between using 'fixed-energy' focusing and deliberate defocusing obtained by adjusting quadrupoles QMA1QMA3; the corresponding beam envelopes are shown in Fig. 11. Defocusing the beam at the degrader induces larger emittance increase (as examined by Flanz et al. [24]) and here results in the desired reduction of transmission (from around 5\% to 3\%). This detuning procedure is part of how the intensity compensation is achieved. 

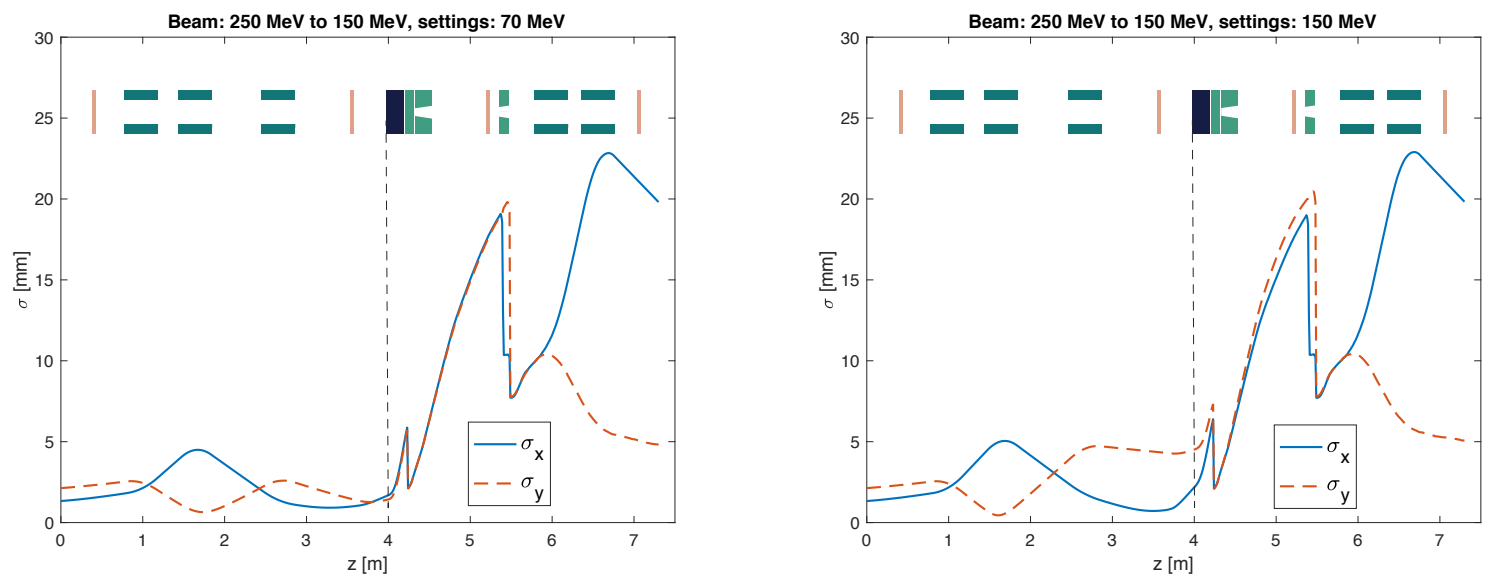

Figure 11: Horizontal (solid blue) and vertical (dashed orange) r.m.s. beam sizes along the beamline for $150 \mathrm{MeV}$ protons downstream of the degrader. (Left) QMA1-QMA3 set for $70 \mathrm{MeV}$; (right) QMA1-QMA3 set to defocus the beam spot at the degrader. For a detailed beamline description see Fig. 3 The vertical dashed line shows the position of the degrader upstream face.

Table 6: Transmission and r.m.s. emittance, at the degrader exit face and at the monitor MMAP11/12, for different energy settings of the beamline elements. The mean proton energy after the multiple-wedge degrader is $150 \mathrm{MeV}$.

\begin{tabular}{llrr}
\hline Location & Parameter & $\begin{array}{r}\text { Setting: } 70 \mathrm{MeV} \\
\text { (this study) }\end{array}$ & $\begin{array}{r}\text { Setting: } 150 \mathrm{MeV} \\
\text { (operation) }\end{array}$ \\
\hline \multirow{2}{*}{ Degrader exit } & Transmission [\%] & $68.03 \pm 0.01$ & $63.62 \pm 0.01$ \\
& $\epsilon_{x}[\mathrm{~mm}$ mrad] & $63.54 \pm 0.07$ & $67.18 \pm 0.07$ \\
& $\epsilon_{y}[\mathrm{~mm}$ mrad] & $56.44 \pm 0.12$ & $105.03 \pm 0.09$ \\
Transmission [\%] & $4.74 \pm 0.01$ & $3.05 \pm 0.02$ \\
& $\epsilon_{x}[\mathrm{~mm}$ mrad] & $29.08 \pm 1.53$ & $38.40 \pm 0.34$ \\
& $\epsilon_{y}[\mathrm{~mm} \mathrm{mrad}]$ & $18.94 \pm 0.59$ & $20.26 \pm 0.20$ \\
\hline
\end{tabular}




\subsection{Alternative Degrader Designs and Materials}

We discussed earlier that higher-density degrader materials may be advantageous in increasing beam transmission above what might be expected simply from the use of degrader materials with a lower average $Z$. Suitable materials for use in particle therapy facilities should be of low toxicity and give low neutron production under proton bombardment; they should also be manufacturable with good precision, have reasonable cost, and be physically robust. Low- $Z$ materials that have been considered to replace the more common graphite are beryllium, diamond and boron carbide [12, 17]. Despite it having the smallest $Z$, beryllium is typically discounted because of the hazards involved in manufacturing and breakage to do with beryllium dust inhalation. Boron carbide is favoured at present due to its high density and reasonable cost but it is still quite difficult to sinter during production, leading to relatively poor homogeneity. A composite of boron carbide and graphite has also been proposed as it is denser and of lower average $Z$ than graphite, and also softer than boron carbide and hence easier to process [25, 26]. Depending on the energy range, different designs not only vary the degrader thickness but also the degrader material for a given energy. For example, the HUST design proposes a multiple-material degrader at lower energies, whilst that proposed by IBA utilises a 'wheel' degrader combining the use of aluminium, graphite and beryllium [12]27].

A hybrid degrader consisting of two pairs of graphite wedges and two boron carbide blocks is under investigation at HUST [28]; it is to be mounted directly before the superconducting gantry. For a smaller energy reduction (from $250 \mathrm{MeV}$ to a maximum of $170 \mathrm{MeV}$ ) only the wedges would be used; being lighter (1/4 of the full multi-wedge degrader weight) they allow for higher motion speed. For a larger decrease in the beam energy, a single or double block would be inserted in addition to the wedges providing a higher total transmission (up to $50 \%$ relative transmission increase for the lower energy ranges $70-120 \mathrm{MeV}$ ). A degrader consisting of only multiple wedges was also considered at HUST [25]; whilst the total number of wedges was optimised to be exactly the same as at PSI (2.5 wedges on each side), a $30^{\circ}$ angle of the wedges was proposed. An additional proposal of the non-triangular variable angle of the wedges for different energy reduction was made.

The work of Gerbershagen et al. [11] presented promising results when comparing a block of boron carbide with graphite. However, the work we show here indicates that the degrader geometry is very important to take into account, and must be considered alongside the choice of material when assessing the design of a particle therapy degrader. Our results indicate that a single block (i.e. a rotating wheel offering a number of positions each with a different fixed thickness) is the most beneficial geometry when trying to maximise overall particle transmission, and such geometry should eventually be considered when trying to obtain the highest dose rates at the lowest energies.

\section{Funding}

This project has received funding from the European Union's Horizon 2020 research and innovation programme under the Marie Sklodowska-Curie Grant No. 675265, OMA Optimization of Medical Accelerators. 


\section{References}

[1] J. Verba, W. Kundig, A. Paul, J. Richardson, B. Wright, in: Proceedings of the International Conference on Sector-Focused Cyclotrons and Meson Factories, 1963. [link]. URL http://accelconf.web.cern.ch/AccelConf/c63/papers/cyc63c03.pdf

[2] C. Baumgarten, Cyclotrons with fast variable and/or multiple energy extraction, Phys. Rev. ST Accel. Beams 16 (2013) 100101. doi:10.1103/PhysRevSTAB.16.100101. URL https://link.aps.org/doi/10.1103/PhysRevSTAB.16.100101

[3] C. Hori, T. Aoki, T. Seki, Variable-energy isochronous accelerator with cotangential orbits for proton beam therapy, Nuclear Instruments and Methods in Physics Research Section A: Accelerators, Spectrometers, Detectors and Associated Equipment 922 (2019) 352 - 356. doi:https://doi.org/10.1016/ j.nima.2019.01.005.

URL http://www.sciencedirect.com/science/article/pii/S0168900219300105

[4] M. J. Van Goethem, R. Van Der Meer, H. W. Reist, J. M. Schippers, Geant4 simulations of proton beam transport through a carbon or beryllium degrader and following a beam line, Phys. Med. Biol 54 (2009) 5831-5846. doi:10.1088/0031-9155/54/19/011.

URL http://iopscience.iop.org/article/10.1088/0031-9155/54/19/011/pdf

[5] V. Favaudon, L. Caplier, V. Monceau, F. Pouzoulet, M. Sayarath, C. Fouillade, M.-F. Poupon, I. Brito, P. Hupé, J. Bourhis, J. Hall, J.-J. Fontaine, M.-C. Vozenin, Ultrahigh dose-rate FLASH irradiation increases the differential response between normal and tumor tissue in mice, Tech. rep.

URL www.ScienceTranslationalMedicine.org

[6] D. Reist, H, Doelling, R, Graf, M, Pedroni, E, Schippers, M, Breiing, A, Evertz, J, Schapper, A fast degrader to set the energies for the application of the depth dose in proton therapy. Technical Report Volume V, Tech. rep. (2002).

URL http://www.psi.ch

[7] V. Rizzoglio, Precise Beam Dynamics Models for Transport Lines in a Cyclotron-based Proton Therapy Facility|doi : 10.3929/ethz-b-000280874

URL https://doi.org/10.3929/ethz-b-000280874

[8] M. Daum, Progress of the nucleon area NA1, Tech. rep. (1989).

URL https://www.researchgate.net/profile/Arie\{_\}Taal/publication/ $226542239\left\{_{-}\right\} \operatorname{Muon}\left\{_{-}\right\}$attachment $\left\{_{-}\right\}$in $\left\{_{-}\right\}$prompt $\left\{_{-}\right\}$fission $\left\{_{-}\right\}$of $237 \mathrm{~Np} /$ links/ 5426859b0cf238c6ea779505/Muon-attachment-in-prompt-fission-of237Np.pdf $\{\#\}$ page=6

[9] E. Pedroni, R. Bacher, H. Blattmann, T. Bohrinaer, A. Coray, A. Lomax, S. Lin, G. Munkel, S. Scheib, U. Schneider, A. Tourovskv, The 200-Mev proton therapy project at the Paul Scherrer Institute: Conceptual design and practical realization, Medical Physics 22 (1) (1995) 37-53. doi:10.1118/1.597522.

[10] V. Rizzoglio, A. Adelmann, C. Baumgarten, D. Meer, J. Snuverink, V. Talanov, On the accuracy of Monte Carlo based beam dynamics models for the degrader in proton therapy facilities, Nuclear Instruments and Methods in Physics Research, Section A: Accelerators, Spectrometers, Detectors and Associated Equipment 898 (December 2017) (2018) 1-10. doi:10.1016/j.nima.2018.04.057. URL https ://doi.org/10.1016/j.nima.2018.04.057

[11] A. Gerbershagen, C. Baumgarten, D. Kiselev, R. Van Der Meer, Y. Risters, M. Schippers, Measurements and simulations of boron carbide as degrader material for proton therapy, Physics in Medicine and Biology. URL http://iopscience.iop.org/article/10.1088/0031-9155/61/14/N337/pdf

[12] R. Tesse, A. Dubus, N. Pauly, C. Hernalsteens, W. Kleeven, F. Stichelbaut, Numerical Simulations to Evaluate and Compare the Performances of Existing and Novel Degrader Materials for Proton Therapy, Journal of Physics: Conference Series 1067 (9) (2018) 2-5. arXiv:arXiv:1804.10838v1, doi:10.1088/1742-6596/1067/9/092001.

[13] T. J. Roberts, D. M. Kaplan, G4beamline simulation program for matter-dominated beamlines, Proceedings of the IEEE Particle Accelerator Conference (2007) 3468-3470 doi:10.1109/ PAC.2007.4440461

[14] S. Agostinelli, et al., Geant4 - a simulation toolkit, Nuclear Instruments and Methods in Physics Re- 
search Section A: Accelerators, Spectrometers, Detectors and Associated Equipment 506 (3) (2003) 250-303. doi:10.1016/S0168-9002(03)01368-8.

URL https://linkinghub.elsevier.com/retrieve/pii/S0168900203013688

[15] Anastasia Makarova et al, Comparison of Geant4 multiple Coulomb scattering models with theory for radiotherapy protons $\operatorname{arXiv:1204.4470}$ URL http://arxiv.org/abs/1204.4470

[16] S. . M. Seltzer, J. M. Fernandez-Varea, P. Andreo, P. M. Bergstrom, D. T. Burns, I. Krajcar Bronic, C. K. Ross, F. Salvat, Report 90, Journal of the International Commission on Radiation Units and Measurements 14 (1). doi:10.1093/jicru/ndw043.

URL https://doi.org/10.1093/jicru/ndw043

[17] V. Anferov, Energy degrader optimization for medical beam lines, Tech. rep. (2003). URL http://www.nac.ac.za/

[18] R. Dölling, Profile, current, and halo monitors of the proscan beam lines, AIP Conference Proceedings 732 (1) (2004) 244-252. arXiv:https://aip.scitation.org/doi/pdf/10.1063/1.1831154, doi:10.1063/1.1831154

URL https://aip.scitation.org/doi/abs/10.1063/1.1831154

[19] R. Dölling, Ionisation chambers and secondary emission monitors at the proscan beam lines, AIP Conference Proceedings 868 (1) (2006) 271-280. arXiv:https://aip.scitation.org/doi/pdf/10.1063/ 1.2401414, doi: $10.1063 / 1.2401414$.

URL https://aip.scitation.org/doi/abs/10.1063/1.2401414

[20] D. C. Hall, A. Makarova, H. Paganetti, B. Gottschalk, Validation of nuclear models in geant4 using the dose distribution of a $177 \mathrm{MeV}$ proton pencil beam, Physics in Medicine and Biology 61 (1) (2015) N1-N10. doi:10.1088/0031-9155/61/1/n1. URL https://doi.org/10.1088\%2F0031-9155\%2F61\%2F1\%2Fn1

[21] D. A. Edwards, M. J. Syphers, An introduction to the physics of high energy accelerators, Wiley, 1993. URL https://books.google.co.uk/books?hl=en $\{\&\} l r=\{\&\}$ id=uczGoTbHd98C $\{\&\} \circ i=$ fnd $\{\&\}$ pg=PP2 $\{\&\}$ dq=syphers+introduction+to+particle+accelerators $\{\&\}$ ots $=$ EeSY0fY6as $\{\&\}$ sig=7xq3Klli3EtnvVKf JqidZUe3hfM $\{\&\}$ redir $\left\{\_\right\} e s c=y\{\#\} v=o n e p a g e\{\&\} q=$ syphersintroductiontoparticleaccelerators $\{\&\} f=f$ alse

[22] R. Apsimon, G. Burt, S. Pitman, H. Owen, ProBE-proton boosting extension for imaging and therapy, in: Proceedings of IPAC2016, 2016.

URL https://www.england.nhs.uk/commissioning/spec-

[23] V. Rizzoglio, A. Adelmann, C. Baumgarten, M. Frey, A. Gerbershagen, D. Meer, J. M. Schippers, Evolution of a beam dynamics model for the transport line in a proton therapy facility|doi:10.1103/ PhysRevAccelBeams.20.124702

URL https://journals.aps.org/prab/pdf/10.1103/PhysRevAccelBeams.20.124702

[24] J. B. Flanz, F. Gerardi, E. L. Hubbard, Design considerations for a proton therapy beamline with an energy degrader, AIP Conference Proceedings 392 (1997) 1257. doi:10.1063/1.52693. URL https://doi.org/10.1063/1.52693

[25] Z. Liang, K. Liu, B. Qin, W. Chen, X. Liu, D. Li, Y. Xiong, Design and optimization of an energy degrader with a multi-wedge scheme based on Geant4, Nuclear Inst. and Methods in Physics Research 890 (2018) 112-118. doi:10.1016/j.nima.2018.01.073.

URL https://doi.org/10.1016/j.nima.2018.01.073

[26] H. Wang, Q. Guo, J. Yang, Y. Zhao, X. Wang, Z. Tao, Z. Liu, Z. Feng, L. Liu, Microstructure and thermophysical properties of b4c/graphite composites containing substitutional boron, Carbon 52 (2013) 10 - 16. doi:https://doi.org/10.1016/j.carbon.2012.08.066

URL http://www.sciencedirect.com/science/article/pii/S000862231200718X

[27] V. Jongen, Yves, Poreye, Device for varying the energy od a particle beam extracted from an accelerator (2001).

URL https://patentimages.storage.googleapis.com/11/97/bb/2e0c5fa9f55899/US6433336.pdf

[28] B. Qin, R. Zhao, X. Liu, Design considerations of a superconducting gantry with alternating-gradient 
combined-function magnet (2019).

URL https://indico.cern.ch/event/803528/contributions/3502644/attachments/1902958/ 3142831/Qin_2019Sep4_OMA.pdf 